\title{
An ensemble of future strategies for national infrastructure provision
}

\author{
Adrian J. Hickford ${ }^{1}$, Robert J. Nicholls ${ }^{1}$, Alexander Otto ${ }^{2}$, Jim W. Hall ${ }^{2}$, Simon P. Blainey ${ }^{1}$, Martino Tran², Pranab Baruah ${ }^{2}$ \\ ${ }^{1}$ Faculty of Engineering and the Environment, University of Southampton, Southampton, SO17 1BJ, UK \\ ${ }^{2}$ Environmental Change Institute, University of Oxford, Oxford, OX1 3QY, UK \\ *(corresponding author, e-mail: A.J.Hickford@soton.ac.uk).
}

\begin{abstract}
Well managed, maintained and effective National Infrastructure (NI) systems (i.e. energy, transport, water, waste, ICT) provide the backbone for economic growth and societal wellbeing. Traditionally, NI planning and implementation has been conducted in isolation, based upon fixed assumptions about the future. This fails to recognise both the long-term socio-economic, climate-related and technological challenges and uncertainties of the coming century and the growing interconnectivity between these individual infrastructure systems. Here we develop a methodology for generating a range of strategies for the provision of NI suitable for an integrated 'system-of-systems' assessment of NI's future management. To provide coherent links with policy choices, integrated strategies are developed at an aggregate level with a diversity of investment requirements, demand management techniques, and levels of environmental targets. To facilitate implementation within NI simulation models, the example strategies are constructed from sectoral sub-strategies focusing on changes in demand, capacity utilisation and capacity expansion. Further new strategies can be explored and the approach lends itself to an iterative analytical approach, potentially capturing a wide range of policy questions. It is illustrated using the future provision of Great Britain's transport infrastructure as a case study, and demonstrates how cross-sector strategies for infrastructure provision can be developed.
\end{abstract}

\section{Introduction}

A nation's economic prosperity and societal wellbeing relies on high-quality infrastructure, particularly those essential services and systems which transport key resources and enable global communications (i.e. energy, transport, water, waste and information and communication technologies (ICT)) [1]. Provision of resilient, effective National Infrastructure (NI) systems has become a focus of many advanced economies [1-7], but such systems face a number of serious challenges: (i) an increased demand for infrastructure services from a growing and ageing population; (ii) significant investment requirements to counter the vulnerabilities, capacity limitations and supply insecurities associated with an ageing infrastructure system; (iii) the increasing complexity, diversification and interdependence of infrastructure networks; and (iv) a widespread desire to maintain and improve environmental standards, including decarbonisation across infrastructure sectors.

Overcoming these multiple challenges requires a long-term strategic view on infrastructure provision, especially given the long lifespan of many physical infrastructure assets (particularly in water, transport and energy), and the long lead-time necessary to effect change in these systems $[2,4,8,9]$. However, the feasibility of such long-term planning is, in turn, challenged by future uncertainties associated with demographic, economic, environmental and political changes, as well as uncertainties about the nature of technological change [10], all of which are likely to have a significant effect on the demands and requirements of NI systems. Lastly, there is a wide range of potential future strategies (i.e. choices) concerning the future provision of national infrastructure. 
Historically, policies and decisions regarding individual infrastructure sectors have been made through complex systems of governance and often in isolation, with little regard for other interconnected infrastructures. Levels of investment in infrastructure have been influenced by the perceived political and economic importance of individual sectors, and such investments have fluctuated over time [11, 12]. More recently, the importance of taking a long-term and cross-sectoral view of infrastructure provision has been more widely acknowledged in many countries. For example, within the British Government, Infrastructure UK was formed in December 2009 with the specific aim of ensuring a more harmonised long-term vision of infrastructure planning. It promotes a more integrated approach to NI through the development of a National Infrastructure Plan [3, 13, 14], which identifies a broad strategy for meeting the country's infrastructure needs.

In the longer term, such a broad strategy could evolve in a number of different directions, particularly depending on certain policy choices, such as (i) how much investment is available, (ii) the government's level of commitment to mitigating climate change, and (iii) the impact of differing approaches to managing demand for those services that NI helps to deliver. Figure 1 outlines an analytic process to test the long-term impact and effectiveness of a range of instructure provision strategies across a wide range of socio-economic changes, and hence promote more effective decision-making and planning.

Modelling tools are used to simulate current and possible future configurations of the NI system for each sector and the possible interactions between interdependent sectors which have co-evolved spatially. Thus, a multi-sectoral integrative approach for a particular region is appropriate for this framework [15].

Exogenous input variables to these system models represent a range of scenarios of socio-economic and environmental change, while endogenous input variables are used to represent a diverse set of sector-specific strategies for infrastructure provision; these strategies also need to be combined to create plausible cross-sector strategies for the NI system as a whole.

This paper outlines the challenges of developing integrated strategies of future infrastructure provision across multiple interacting infrastructure sectors, and proposes and demonstrates a framework for their analysis. It is organised as follows. First, a brief background of traditional scenario studies is provided, with an explanation of how this has been adapted within a system-of-systems simulation model framework. This is followed by a more detailed presentation of how cross-sector strategies for infrastructure provision can be constructed. The approach is illustrated for NI in Great Britain (GB), with a detailed development of sector-specific strategies for transport, and their positioning within the crosssector strategy framework. This is followed by discussion/conclusions. 


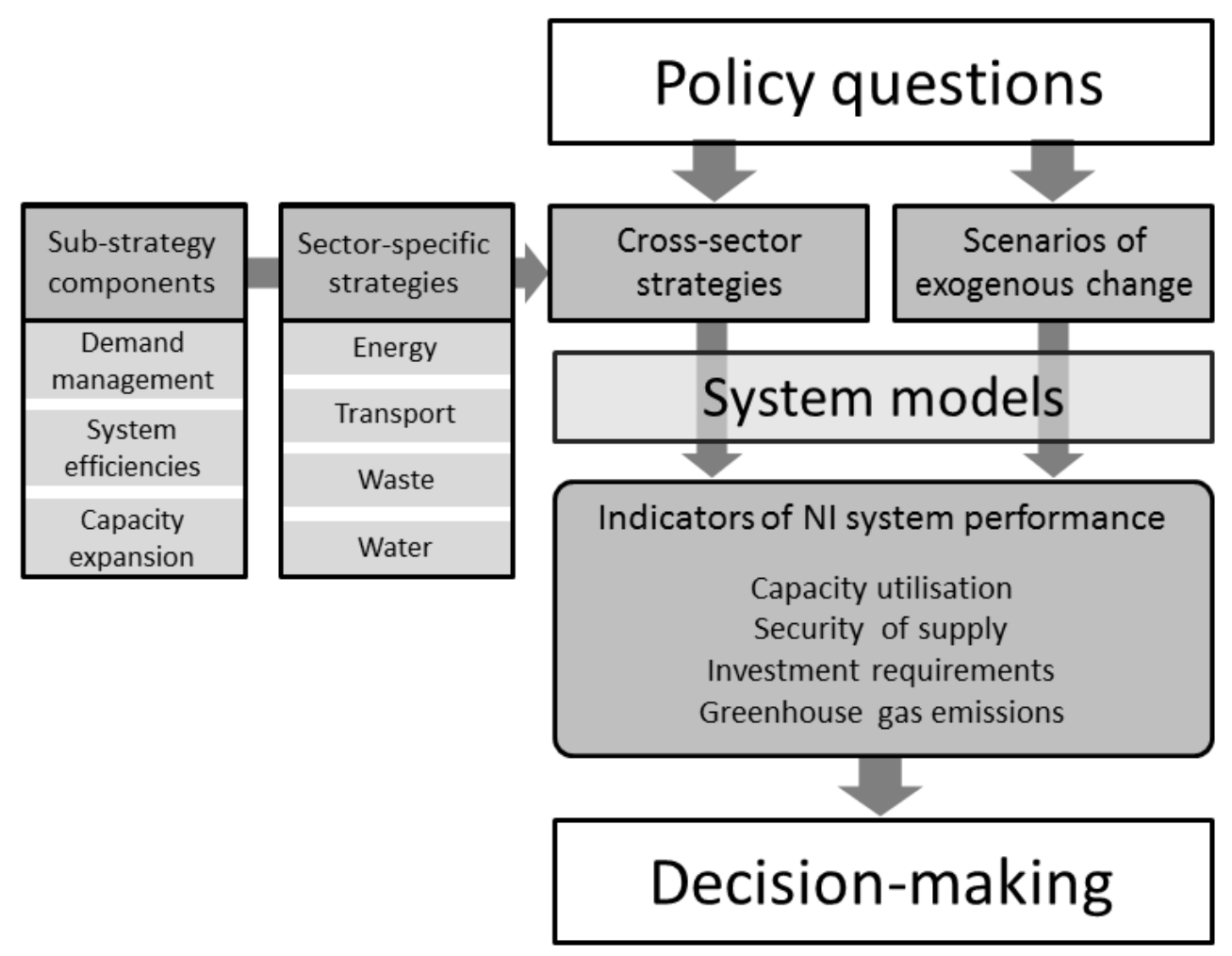

Figure 1: An analytic process using models to inform decision makers about future NI provision

\section{Development of scenarios and strategies for use in a model framework}

One of the traditional tools for assessing uncertain future developments in complex systems is scenario analysis. Both global and regional socio-economic and environmental scenario studies for integrated assessments such as those considered here tend to focus on how fundamental changes to the nature of populations, economies and governance regimes are likely to affect future changes in a particular performance metric of the system. Global Environmental Assessments (GEA), for instance, seek to explore potential impacts and consequences of change in greenhouse gas emissions and resulting climate under differing socio-economic futures or scenarios [16-18].

There are a range of techniques used to develop scenarios, each with particular advantages and disadvantages [19-21]. One common method is to focus on a limited number of scenario families which usually emerge from extremities of a framework defined by contrasting scenario futures (such as the global Intergovernmental Panel on Climate Change (IPCC) Special Report on Emissions Scenarios (SRES) scenarios based on two axes: "Economic" vs. "Environmental" and "Regionalism" vs. "Global integration" [22] and similarly, but at a national scale, those suggested by the Foresight Futures programme in the UK contrasting social values (individuality vs. community) and systems of governance (local autonomy vs. global interdependence) [23]). These scenario approaches have been explicitly linked for the UK in order to apply global scenarios at national and sub-national scales (e.g. [24], [25]).

Some previous studies specifically compare diverse (and sometimes caricatured) options of future policy choices. For example, the impacts of differing strategies for levels of coastal protection under the four SRES scenarios are compared with a 'no protection' 
strategy, which is extremely unlikely to occur, but does offer insight into the benefits of the selected upgraded protection level [26, 27].

However, generally the scenario and strategy generation techniques widely used tend to develop overall visions of some future system state, with a series of distinct versions of the future conditions and policy assumptions; pathways towards each version are identified and assessed. As part of a literature review in the development of the new Shared SocioEconomic Pathways (SSP), van Vuuren et al. [28] expanded on previous scenarios comparisons [18], identifying six differing scenario families shown in Table 1 . These families can each be defined by their basic underlying storylines and assumptions regarding socioeconomic change (i.e. population growth and economic development) and more policyrelated elements (i.e. technological impact, approach to environmental change and governance).

\begin{tabular}{|c|c|c|c|c|c|c|c|}
\hline \multicolumn{2}{|c|}{ Scenario family } & $\begin{array}{l}\text { (1) Economic- } \\
\text { technological } \\
\text { optimism }\end{array}$ & $\begin{array}{l}\text { (2) Reformed } \\
\text { markets }\end{array}$ & $\begin{array}{l}\text { (3) Regional } \\
\text { competition }\end{array}$ & $\begin{array}{l}\text { (4) Regional } \\
\text { sustainable } \\
\text { development }\end{array}$ & $\begin{array}{l}\text { (5) Global } \\
\text { sustainable } \\
\text { development }\end{array}$ & $\begin{array}{l}\text { (6) "Business } \\
\text { as usual” }\end{array}$ \\
\hline \multicolumn{2}{|c|}{$\begin{array}{l}\text { Main societal } \\
\text { objectives }\end{array}$} & $\begin{array}{l}\text { Economic } \\
\text { growth }\end{array}$ & Multiple goals & Security & $\begin{array}{l}\text { Local } \\
\text { sustainability }\end{array}$ & $\begin{array}{l}\text { Global } \\
\text { sustainability }\end{array}$ & $\begin{array}{l}\text { Not explicitly } \\
\text { defined }\end{array}$ \\
\hline \multirow{5}{*}{ 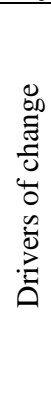 } & $\begin{array}{l}\text { Population } \\
\text { growth }\end{array}$ & Low & Low & High & Medium & Low & Medium \\
\hline & $\begin{array}{l}\text { Economic } \\
\text { development }\end{array}$ & Very rapid & Rapid & Slow & $\begin{array}{l}\text { Ranging from } \\
\text { mid to rapid }\end{array}$ & $\begin{array}{l}\text { Ranging from } \\
\text { slow to rapid }\end{array}$ & $\begin{array}{l}\text { Medium } \\
\text { (globalisation) }\end{array}$ \\
\hline & $\begin{array}{l}\text { Technological } \\
\text { development }\end{array}$ & Rapid & Rapid & Slow & $\begin{array}{l}\text { Ranging from } \\
\text { mid to rapid }\end{array}$ & $\begin{array}{l}\text { Ranging from } \\
\text { mid to rapid }\end{array}$ & Medium \\
\hline & $\begin{array}{l}\text { Environmental } \\
\text { protection }\end{array}$ & Reactive & $\begin{array}{l}\text { Both reactive } \\
\text { and proactive }\end{array}$ & Reactive & Proactive & Proactive & $\begin{array}{l}\text { Both reactive } \\
\text { and proactive }\end{array}$ \\
\hline & $\begin{array}{l}\text { Policies and } \\
\text { institutions }\end{array}$ & $\begin{array}{l}\text { Policies create } \\
\text { open markets }\end{array}$ & $\begin{array}{l}\text { Policies reduce } \\
\text { market failures }\end{array}$ & $\begin{array}{l}\text { Strong } \\
\text { national } \\
\text { governments }\end{array}$ & $\begin{array}{l}\text { Local steering, } \\
\text { local actors }\end{array}$ & $\begin{array}{l}\text { Strong global } \\
\text { governance }\end{array}$ & Mixed \\
\hline
\end{tabular}

Vuuren et al. [28])

Within each of these families, there are certain assumptions associating specific levels of socio-economic change with particular policy-related dimensions. Applied to simulation models of NI, each family could be represented by a single set of input variables. This relatively inflexible approach does not allow assessment of the effectiveness of a range of differing policy dimensions across diverse socio-economic and environmental scenarios. In other words, these approaches do not provide policymakers with the tools to appraise the range of options at their disposal. To enable such appraisal, it is necessary to assess two sets of input variables, one representing the range of scenarios of socio-economic and environmental change, the other representing change resulting from each of the alternative strategies for infrastructure provision. The scenario space evolves from the exogenous socioeconomic and environmental variables, while cross-cutting overall strategic narratives emerge from descriptions of a set of different possible policy approaches and attitudes towards infrastructure provision and changes to the infrastructure system.

Borjeson et al. [29] created a typology of three main techniques for scenario generation based on a classification of three different types of futures - the probable, possible, and preferable futures. Thus, scenario studies tend to fit into three main categories: Predictive, Explorative and Normative. Normative scenarios are target-led; future pathways to reach specific targets are identified, either by cost-effectively preserving and adapting the current situation, or through more investment-intensive transformational means. Predictive scenarios 
aim to predict what will happen in the future, either through narrow, relatively short-term forecasts of the most likely developments, or broader 'what-if' scenarios, groups of forecasts conditional on some specified situation. The Explorative scenario technique aims to explore a wide range of possible situations or developments, usually derived from different viewpoints with a long time horizon. There are two components to this technique: external scenarios which focus on factors beyond the control of the relevant actors; and strategic scenarios, which incorporate various policy options.

The Explorative scenario and strategy assessment is most appropriate for assessing policy options, since it is possible to distinguish the exogenous change variables (population, economy, climate) from those that are policy-related. A range of alternative futures and trajectories of socio-economic change can be generated, mapping a 'possibility space'. Modelling results for a number of diverse strategies can be tested for robustness within this possibility space [30,31]. This approach closely matches the analytic process shown in Figure 1; an example of a model framework suitable for this approach is now presented and illustrated.

\section{Overview of the model framework}

The overall assessment framework incorporates a number of stages, as follows: (1) the generation of ensembles of future scenarios (i.e. plausible situations) of socio-economic and environmental conditions; (2) the generation of a range of interesting, distinct and plausible strategies for future NI provision; (3) the simulation of future demand and capacity in a suite of single-sector simulation models, linked to a central infrastructure database; and (4) the evaluation of infrastructure service performance and the uncertainty analysis or robustness of performance of the varying NI strategies across the scenario space, including interdependencies between sectors [15]. This paper focuses on step (2) above, the generation of a range of national strategies for NI provision, (i.e. policy scenarios) within the overall assessment framework.

In this example, the NI sectors are represented by a suite of soft-coupled models, which estimate capacity of and demand for infrastructure services. The soft-coupling allows an iterative approach where the input variables for one sector model are dependent on the outputs from another sector. The network representations of the systems of physical infrastructure assets used to derive capacities are common to all the sector models, while the method used to derive demand for NI services is dependent on the model structure ${ }^{1}$.

Policy decisions that might change the system state are introduced by strategy input variables representing societal change (incorporated as changes in demand), technological change (incorporated as changes in efficiencies and technology-cost parameters), and systemic change within the physical system of infrastructure assets (incorporated in the configuration and capacity of infrastructure networks). Changes in exogenous variables that influence the performance of the NI system are represented by scenarios of socio-economic

\footnotetext{
${ }^{1}$ For this assessment, the energy sector's capacity model represents GB's electricity and gas generation and supply networks (an expansion of the CGEN modelling and optimisation tool [32]), while demand is estimated using a separate disaggregated demand module [33]; for transport, a national strategic model of trunk road, rail, port and airport infrastructure gives the capacity at regional resolution, while demand is derived through elasticity-based relationships with explanatory variables [34]; a water resources system model is used, coupled with a model of wastewater treatment facilities; and a national solid waste assessment model gives the outputs for the waste sector [35].
} 
variables, climate variables and other technological variables, which are outside the influence of infrastructure planners and investors, and which are assumed to independent of the performance of infrastructure itself. However, while such an assumption is necessary for this 'feed-forward' simulation framework, there are likely to be feedbacks between the NI system performance and the socio-economic background; this separation of external factors and strategies will necessarily lead to some inconsistencies, which have to be considered during the analyses.

NI system performance can be evaluated by assessing the various model outputs across a number of performance indicators, including:

- capacity of and demand for infrastructure services,

- capacity utilisation and security of supply,

- side effects of service provision (greenhouse gas emissions, safety, quality of services, environmental quality), and

- costs for service provision and investments.

This approach provides a generic framework for analysis of long-term change in capacity and demand of NI as interdependent systems in an uncertain future. Rather than reporting on optimal strategies, it allows investigation of the robustness of NI strategy performance across the range of uncertain future conditions. This approach is equivalent to a robust decision analysis of these geographically explicit national-scale model results [36]. It not only enables the identification of capacity constraints in space and time, at which external stresses cause the collapse of service provision performance, but also of levers and links for critical investments to ensure robust and secure supply of infrastructure services. With this capability, such a suite of NI analysis tools can provide the basis of an evidence-based vision for a NI system in the 21st century.

\section{Scenario generation}

The first step of the modelling framework is to develop scenarios of future socioeconomic and environmental change. At a national scale, population change and economic growth are the main drivers of change in demand for NI services [35]; the demand effects of these socio-economic drivers are modified by technological innovation and behavioural change. A range of futures comprising these socio-economic components can be assessed as part of the model framework. In the Explorative scenario approach, four components, assumed to be exogenous to the analysis, make up the external scenarios: (i) demographic changes, (ii) changes to both national and global economies, (iii) global fossil-fuel costs, and (iv) climate change. The framework includes development of a range of internally consistent socio-economic future trajectories derived by combining these socio-economic components, acknowledging the interaction between prosperity and population growth [37]. An ensemble of scenarios of regional economic change per economic sector (as well as a series of other economic variables) has been developed to 2050 (and extrapolated to 2100 for longer term analyses), based on the demographic projections and on assumptions about future technological change and world-economic conditions. Further outputs, based on possible future global economic conditions, include estimates of the long term effect on fossil-fuel costs.

Each of these four components affects the way infrastructure services are used, either in terms of the level of demand, the capacity provision, or the cost implication (which in turn 
affects demand). The four components are now considered in turn, using Great Britain as an example.

(i) Demographic change largely affects demand for infrastructure services. A range of long-term projections of population change across the UK (of which GB is a part) is published by The Office of National Statistics (ONS). The ONS projections have been extended to the year 2100, based on the most recently available mid-year population estimates and a set of underlying demographic assumptions regarding fertility, mortality and migration, which are in turn products of a range of economic, political and social forces, bounded by upper and lower limits to reflect future uncertainties [38]. These projections can be further developed into a range of spatially disaggregated demographic scenarios, giving a population range of 60-140 million for GB in 2100 [37].

(ii) Economic change affects the population's ability to utilise infrastructure services; higher GDP is likely to result in higher demand for NI, as people tend to consume more energy and travel further and more often. Economic growth may also affect governmental and NI owners' ability to invest in infrastructure systems, although such investment may be seen as vital to ensure economic growth [39-42].

(iii) Global fossil-fuel costs affect operating costs and transport costs in particular. National policy measures such as carbon taxes may affect these costs, but these are generally assumed to be exogenous to the models.

(iv) Climate change particularly affects resource availability for Water. Scenarios for future projected UK climate change (seasonal temperatures, rainfall, sea-level rise) are given by UKCP09 [43]. These are in turn based on the IPCC global AR4 assessment [44] which uses the SRES scenario framework ${ }^{2}$ [45]). These projections are being superseded by a new scenario framework based on the on-going work on Representative Concentration Pathways (RCPs) [46] and associated Shared Socioeconomic reference Pathways (SSPs) [47]. However, while the new global scenarios are available [48], it will be some time before new downscaled UK climate change scenarios are made available. Furthermore, assessments based on the SRES framework can be reinterpreted into the RCP framework as need be.

\section{Strategy generation}

The next step in the modelling framework is to develop a variety of sector-specific strategies for national infrastructure provision which can be assessed for the range of socioeconomic and environmental scenarios. The 'strategy' aspect of the Explorative approach described above requires the development of a range of diverse sectoral and cross-sectoral, long-term national infrastructure provision strategies. A NI strategy comprises a series of system interventions through regulation, investment, taxation, or incentives to change demand for and capacity of infrastructure service provision. This representation of a NI strategy allows inclusion of specific elements of real-world policies regarding infrastructure provision, as well as analysis of the robustness of a range of such policies.

${ }^{2}$ In UKCP09, projections are developed under three different emissions scenarios, two of which come from the A1 storyline, and one from the B1 storyline developed by the IPCC Special Report on Emissions Scenarios (SRES). Within UKCP09 the emission scenarios are labeled based on their relative greenhouse gas emissions levels - High (SRES A1FI), Medium (SRES A1B) and Low (SRES B1) - and comprise a wide range, but not the full set of SRES emissions scenarios. 
The framework described here uses a strategy generation technique comprising a multistep hierarchical process that combines a top-down approach from relevant real-world policy questions with a bottom-up approach from the input variables of the NI models of capacity and demand. This requires a hierarchical representation of policy options. From a top-down perspective, as presented in Figure 2, this consists of (i) high-level policy dimensions; (ii) a range of sub-strategy components representing changes to the structure and use of infrastructure systems (in terms of demand management, system efficiencies and capacity expansion); (iii) the combination of these sub-strategy components into sector-specific strategies; and (v) model implementation of these strategies. Each additional step of the hierarchy adds detail to the strategy description. The same hierarchy is also used in a bottomup approach to classify and categorise a set of high-level sector-specific strategies of infrastructure provision created by generic combinations of low-level options, or even of model input variables.

At a high level, policymakers wish to explore the implications of different scales of both public and private investment. At the same time, there are different possible levels of ambition for environmental targets for NI. Differing strategies will have varying implications for how demand is managed, either through changes in costs to the consumer, changes in technologies (including the rate and diversity of technological innovation) or the impacts of changing land use factors. This range of policy dimensions has been selected as a result of discussions with government, industry and other stakeholders.

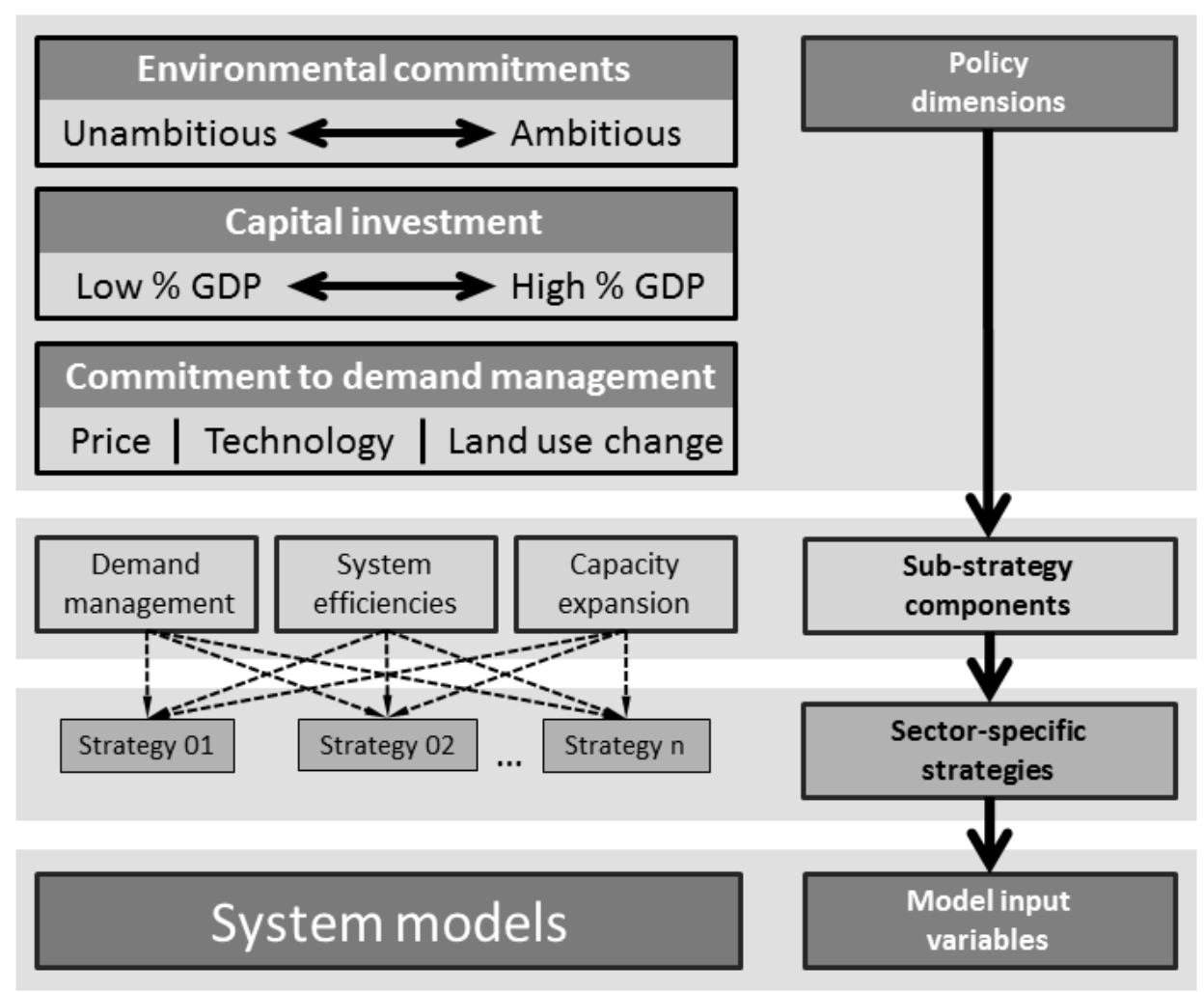

Figure 2: The top-down strategy generation process, from high-level policy dimensions (environmental ambition, capital investment level, demand-related policy focus), through components of infrastructure change (demand management, system efficiencies, capacity expansion) and distinct sector-specific strategies, to interpretations of types of sectoral change as inputs to the models 
The high-level cross-sectoral policy dimensions discussed above are not easy to implement in NI models of capacity and demand. However at the next hierarchical level, a set of sub-strategic components emerge from specific policy decisions which aim to influence (a) changes in demand through social and behavioural change, and (b) changes in capacity utilisation and provision either by technologically increasing system efficiencies or through physical changes to the suite of infrastructure assets. These components can then be combined to create sector-specific infrastructure provision strategies which are more readily ascribed to endogenous variables within the models. These sub-strategic components are now considered.

\section{a) Sub-strategies for demand management}

Consumer demand for NI services can be influenced by changing consumer behaviour, either through education, pricing structures and incentives, or new consumer technologies.

Specific targeted information can affect overall use of infrastructure services: energy saving schemes can reduce domestic energy requirements; societal and environmental pressure can promote modal shift away from private vehicles to more sustainable modes, as well as influencing change in waste recycling and other resource recovery; local levels of grey water recycling could be increased by introducing water usage schemes (e.g. metering). Such measures are attractive in terms of the likelihood of lower overall costs of implementation, especially if subsequent levels of societal behaviour change are not insignificant.

Behavioural change can be effected through a number of solutions. Pricing measures, such as taxation and financial incentive policies, could have greater influence on behaviour change. Road user charging measures, or other regulations or taxes designed to reduce fossil fuel use, such as preferential tariffs for electric vehicles, could change travel behaviour as well as the overall make-up of the vehicle fleet; tax incentives could encourage investment in new technologies; and volume-based tariffs for water consumption or waste generation could reduce the requirements for water and waste treatment. While these behavioural interactions are complicated in the real world, they are necessarily represented relatively simplistically in the models by modifying the relevant input variables.

Demand can also be influenced by technological changes which alter the way in which a system is used. For example increased energy efficiency in domestic appliances, alongside the national roll-out of smart meters is likely to influence energy demand, and increased use of ICT could result in changes in travel habits, including the substitution of virtual meetings for some business trips.

\section{b) Sub-strategies enabling change in capacity utilisation and provision}

Technological advances and different approaches to capacity utilisation can affect the overall efficiency of an infrastructure system. For example, efficiencies in road transport can be achieved through increased fuel economy, optimised route planning or vehicle-to-vehicle and vehicle-to-grid interactions. Losses in energy and water distribution could be reduced through new technologies enabling real-time monitoring of the distribution systems.

Structural changes to the infrastructure system itself will be achieved through newbuild (such as new rail and road links, power stations, reservoirs, water treatment works, or recycling provision), and adaptation of existing infrastructure by replacing outdated infrastructure with modern materials or incorporating new technologies. The transition to 
renewable energy generation is one example of how the physical infrastructure required for distribution of energy may remain relatively unchanged while the landscape of options for energy generation changes significantly.

\section{Defining and combining sector-specific infrastructure provision strategies}

A number of sector-specific infrastructure provision strategies have been developed which represent a menu of plausible options within each infrastructure sector. These strategies are constructed from combinations of sub-strategy components focused on changes in demand, infrastructure composition and supply as described above, spanning the range of policy dimensions shown in Figure 2. The number of strategies for each sector has been deliberately limited to between five and ten. While this is more than most of the traditional scenario studies discussed above, it is still much smaller than the number of conceivable strategies, but for computational feasibility, it is desirable to limit the number of combinations of sub-strategies. In the first instance, the emphasis has been put upon developing a reasonably concise set of distinct strategies spanning the range of possibilities for each sector, which can subsequently be combined to create contrasting cross-sectoral strategies.

\subsection{An example: Transport strategy composition and narratives}

In this section, we describe in greater detail the infrastructure provision strategies that have been developed for the transport sector to illustrate these components and their relationship. These are then positioned within a cross-sector strategy framework consisting in this instance of four contrasting strategies.

Demand- and capacity-based sub-strategy components for each sector are developed under the three headings of demand management, changes in capacity utilisation and changes in the physical structure of the system. Each of the sub-strategy components has a range of underlying assumptions about how the change will be effected as explained below for the transport sector, in which a total of 12 sub-strategy components are defined to consider the range of possible policy directions in a manageable set. By combining the transport sub-strategy components (Demand TD, Capacity TC, and Structure TS), overarching transport infrastructure provision strategies (TIPS) can be constructed. The set of selected strategies is summarised in Table 2 and described in detail below. All the underlying sub-strategies are used at least once, and in one case they are combined. While this is only a small subset of all possible combinations, the selection has resulted in a range of strategies each with a distinct narrative; where feasible, evidence for the model implementation is also described. These are adapted from narratives originally set out in Blainey et al. [49]. The strategies have been assigned iconic titles, but it should be noted that such titles could also apply to other combinations of sub-strategies. 


\begin{tabular}{|l|l|l|l|}
\hline \multirow{2}{*}{$\begin{array}{l}\text { Transport infrastructure } \\
\text { provision strategy }\end{array}$} & \multicolumn{3}{|l|}{ Sub-strategies } \\
\cline { 2 - 4 } & $\begin{array}{l}\text { Demand } \\
\text { management }\end{array}$ & $\begin{array}{l}\text { Capacity } \\
\text { utilisation }\end{array}$ & $\begin{array}{l}\text { Physical } \\
\text { structure }\end{array}$ \\
\hline TIPS0: 'Decline and Decay' & TD0 & TC0 & TS0 \\
\hline TIPS1: 'Predict and Provide’ & TD1 & TC1 & TS1 \\
\hline TIPS2 ‘Cost and Constrain’ & TD2 & TC0 & TS2 \\
\hline TIPS3 ‘Adapting the Fleet' & TD0 & TC2 & TS3 \\
\hline TIPS4 ‘Promo-Pricing' & TD3 & TC0 & TS3 \\
\hline TIPS5 ‘Connected Grid' & TD4 & TC2 & TS2/TS3 \\
\hline TIPS6 ‘Smarter Choices' & TD2 & TC2 & TS2 \\
\hline
\end{tabular}

Table 2: A summary of transport infrastructure provision strategies and their component sub-strategies.

\section{TIPS0: 'Decline and Decay'}

This 'minimal-intervention' strategy describes a future where no replacements are found for fossil fuels, there is no investment in infrastructure beyond that required to maintain the status quo, and no further technological innovation in transport takes place. There is a growth in the use of slow but fuel-efficient modes (walk and cycle for passengers, canals/coastal shipping for freight) and in public transport. The components of this provision strategy have been specifically selected to create an example strategy with small levels of change in demand management, capacity utilisation and the physical infrastructure; while it provides an example of an 'unrealistic' alternative future, it nevertheless allows comparison of the other strategies with a 'do minimum' baseline. The strategy includes the following substrategy components:

- TD0: minimal change in per capita demand - The minimal-intervention 'baseline' whereby current trends in demand are assumed to continue.

- TC0: minimal change to capacity utilisation - The minimal-intervention 'baseline' whereby current trends in capacity utilisation are assumed to continue.

- TS0: minimal structural change - The minimal-intervention 'baseline'; future investment for new roads, rail and airports is not forthcoming, so the current transport network remains largely unchanged.

TIPS1: 'Predict and Provide'

Under this strategy demand modelling drives infrastructure construction, echoing the road policy followed in the UK during the 1970s and 1980s. The model automatically 'builds' additional infrastructure when nodal or link-based capacity utilisation reaches a particular limit, and these capacity enhancements are assumed to become effective the following year. While such infrastructure construction is not directly dependent on any other transport-related factor, technological innovations for established modes are assumed to continue, and fuel efficiency improvements are therefore modelled based on external evidence for road [50], rail [51], air [51] and sea transport [52]. Take up of alternative fuels for road vehicles is assumed to be at the lower end of the range [52-56]. Rail electrification is assumed to occur at a moderately fast rate, with 200 track km per year electrified after 2021. The effects of these changes in fuel consumption and type on travel demand are captured via their effect on the model cost variables. This provision strategy includes elements of the following sub-strategy components: 
- TD1: unconstrained growth in per capita demand - Higher per capita transport demand, particularly for private modes and air. There is an implication of no overarching demand management intervention.

- TC1: less efficient capacity utilisation - Lack of intervention results in the use of the transport network becoming generally less efficient, as there is more congestion on roads, rail and at airports. This is likely to result from a dearth of technological solutions to current problems or from insufficient construction of additional infrastructure.

- TS1: widespread expansion - There is a major focus on the expansion of the system, through large scale road building or widening, additional railway lines, and airport and seaport expansion.

TIPS2: 'Cost and Constrain'

This strategy models the use of congestion pricing to suppress demand at capacityconstrained nodes and links for all modes. Pricing varies over time and space and between vehicle types (for road traffic) with maximum charges set to be equivalent to the non-variable costs for particular modes and vehicle types. A national workplace parking levy is also introduced. A moderate level of take-up of alternative fuels for road vehicles is assumed, alongside improvements in fuel efficiency. Rail electrification also occurs at a moderate rate (100 track km per year), and a small amount of additional road, rail and airport infrastructure is constructed. This provision strategy includes elements of the following sub-strategy components:

- TD2: managed decline in per capita demand - Per capita demand for car use decreases as use of public transport and levels of cycling and walking increase, managed through either pricing or behaviour change measures.

- TC0: minimal change to capacity utilisation - The minimal-intervention 'baseline' whereby current trends in capacity utilisation are assumed to continue.

- TS2: limited expansion - Only limited funding is available for infrastructure expansion, meaning that new construction focuses on pinch-points and bottlenecks in the current transport system.

TIPS3: 'Adapting the Fleet'

This strategy envisages rapid technological development driving a high level of take-up of alternative vehicle fuels, along with major improvements in road vehicle fuel efficiency. Similar improvements are seen in fuel efficiency for other modes, and rail electrification occurs at a rapid rate (300 track km per year). Rail journey times are assumed to be reduced slightly, and maximum aircraft capacities are increased by $20 \%$ by 2050 as a result of the use of lighter construction materials. A moderate amount of additional infrastructure is constructed. This provision strategy includes elements of the following sub-strategy components:

- TD0: minimal change in per capita demand - The minimal-intervention 'baseline' whereby current trends in demand are assumed to continue.

- TC2: more efficient capacity utilisation - There is an increased efficiency in the use of the transport network, as technological advances allow autonomous vehicles to travel closer and at faster speeds, and more sophisticated rail and airport capacity optimisation methods are used.

- TS3: network electrification - In combination with other structural changes, there is widespread electrification, both in rail and road vehicles. There are significant energy demand interdependencies associated with this strategy. 


\section{TIPS4: 'Promo-Pricing'}

In addition to congestion charging, this strategy also sees the introduction of emissionsbased pricing. This is accompanied by a high level of take-up of alternative (low emission) vehicle fuels, along with fuel efficiency improvements. The strategy also includes a moderate quantity of infrastructure construction, along with rapid rail electrification. Aircraft load factors are assumed to increase as a result of the highly disaggregated pricing policy, but maximum capacities remain unchanged. This provision strategy includes elements of the following sub-strategy components:

- TD3: spatial redistribution of demand - No overall change in demand across the transport system as a whole, but there are more localised changes in where and when transport demand occurs, either through 'peak spreading' (i.e. a broadening of traffic flow profiles in peak periods) or redistribution of higher flows across the wider network. These changes could be resultant from changes to work patterns, or the influence of smart GPS route guidance systems.

- TC0: minimal change to capacity utilisation - The minimal-intervention 'baseline' whereby current trends in capacity utilisation are assumed to continue.

- TS3: network electrification - In combination with other structural changes, there is widespread electrification, both in rail and road vehicles. There are significant energy demand interdependencies associated with this strategy.

\section{TIPS5: 'Connected Grid'}

Developments in ICT and its application to transport see maximum capacities increased on both roads and railways through the introduction of advanced control systems. There is a high level of take-up of alternative road fuels, along with large improvements in vehicle fuel efficiency. The Gross Value Added (GVA) elasticities in all models are progressively reduced by up to $50 \%$ over the study period, to reflect the negative impact of ICT improvements on the propensity to travel. As this strategy focuses on technology, only a small amount of additional infrastructure is constructed. This provision strategy includes elements of the following sub-strategy components:

- TD4: ICT replacement for demand - Per capita demand reduces due to the increasing influence of information and communication technologies (ICT) on travel behaviour. One example is the use of virtual environments for business meetings.

- TC2: more efficient capacity utilisation - There is an increased efficiency in the use of the transport network, as technological advances allow autonomous vehicles to travel closer and at faster speeds, and more sophisticated rail and airport capacity optimisation methods are used.

- TS2: limited expansion - Only limited funding is available for infrastructure expansion, meaning that new construction focuses on pinch-points and bottlenecks in the current transport system.

- TS3: network electrification - In combination with other structural changes, there is widespread electrification, both in rail and road vehicles. There are significant energy demand interdependencies associated with this strategy.

TIPS6: 'Smarter Choices'

A range of 'soft' interventions are introduced in this strategy, including smarter choices, smart logistics and urban freight innovation schemes. These are all assumed to lead to a $10 \%$ reduction in relevant road traffic, and are accompanied by a high level of take-up of 
alternative fuels and moderate levels of rail electrification and vehicle efficiency improvements. Again, only a small amount of additional infrastructure is constructed. This provision strategy includes elements of the following sub-strategy components:

- TD2: managed decline in per capita demand - Per capita demand for car use decreases as use of public transport and levels of cycling and walking increase, managed through either pricing or behaviour change measures.

- TC2: more efficient capacity utilisation - There is an increased efficiency in the use of the transport network, as technological advances allow autonomous vehicles to travel closer and at faster speeds, and more sophisticated rail and airport capacity optimisation methods are used.

- TS2: limited expansion - Only limited funding is available for infrastructure expansion, meaning that new construction focuses on pinch-points and bottlenecks in the current transport system.

\subsection{Developing cross-sector strategies}

In order to assess the impact across all sectors of different approaches to infrastructure provision, cross-sector strategies can be developed by combining specific transport strategies with infrastructure provision strategies from other sectors (particularly energy) with similar underlying assumptions. Four such contrasting strategies are as follows:

a) Minimum Intervention (MI) - taking a general approach of minimal intervention, reflecting historical levels of investment, continued maintenance and incremental change in the performance of the current system (e.g. TIPS0: Decline and Decay). There is no long-term vision to reduce future demand or implement more stringent commitments to environmental policies; instead, the focus is on short-term incremental improvements at a sector level, and thus fails to account for sectoral interdependencies.

b) Long-term Capacity Expansion (CE) - focusing on planning for the long term by increasing investment in the near future to avoid capacity constraints in the longer term. (e.g. TIPS1: Predict and Provide). Priority is given to the expansion of physical capacity to alleviate pinch-points and bottle-necks soon after they are identified, which may be effective at meeting demand in the short to medium-term, but is likely to perform poorly in the longer term due to (i) physical limitations in capacity expansion, (ii) lock-in to current technologies and design, and (iii) no long-term vision to reduce or redistribute demand.

c) Increasing System Efficiency (SE) - focusing on deploying the full range of technological and policy interventions to optimise the performance and efficiency of the current system targeting both supply and demand (e.g. TIPS2: Cost and Constrain, TIPS3: Adapting the Fleet and TIPS4: Promo-Pricing). This implies targeted investments to increase capacity at severe bottlenecks in the shorter term, but the medium- to long-term vision is to invest heavily in maximising the throughput of the current system, without high investments in capacity expansion.

d) System Restructuring (SR) - focusing on fundamentally restructuring and redesigning the current mode of infrastructure service provision, deploying a combination of targeted centralisation and decentralisation approaches (e.g. TIPS5: Connected Grid and TIPS6: Smarter Choices). This will require long-term investments aiming to utilise a wide range of technological innovation, incorporating policy incentives and integrated planning and design. 
Subsequent assessment of these cross-sector strategies can be used to compare and contrast the total system performance of national infrastructure, particularly in terms of cumulative investment, and environmental performance (in terms of carbon emissions). These metrics can be normalised and aggregated across space, time or sectors allowing for comparative analysis of current and future performance between cross-sector strategies. The strategies are designed to be contrasting and distinct, although there will inevitably be some overlap between them. Also, the relatively simplistic nature of this cross-sector assessment does not necessarily take account of the interdependencies and feedbacks inherent in such a complex system, although such feedbacks can be considered to be one-way dependencies assessed via a small number of iterations steps of the whole framework. Other sectoral and cross-sectoral strategies can be developed as needed, including strategies developed as a response to policy needs, and learning from the initial analysis in an iterative manner.

\section{Discussion and conclusions}

Integrated assessments have been applied widely to analyse complex social, economic, technical and environmental processes and their interactions [57]. However, while the different drivers have often been analysed in some detail, the potential effect of policy interventions has usually been treated in a rather simple caricaturised manner. This provides useful insights on the broad sensitivities to contrasting policy directions, but it does not allow the investigation of the more detailed strategies that are relevant to issues such as national provision of infrastructure as considered by this paper.

Whilst there have been several calls for a strategic approach to infrastructure provision in Great Britain and elsewhere in the world, long-term strategies that take account of crosssectoral interdependence have yet to be specified. We argue that this absence of adaptive strategies is in part due to methodological limitations. This paper has sought to address that lacuna.

A first attempt at developing such strategies is described by Hall et al [9] as part of the Infrastructure Transitions Research Consortium (ITRC) Fast Track Analysis (FTA). Three distinct cross-sectoral transition strategies were developed: two which focused on the availability of investment (a "Capacity-Intensive" strategy, which assumed high investment in new capacity to maintain supply security, and a "Capacity-Constrained" strategy, in which no increases to current levels of infrastructure investment were envisaged) and one which focused on a reorientation of infrastructure provision (a "Decentralised" strategy). However, it was difficult to link these strategies to the wide range of policy choices that are available across the range of infrastructure sectors that were being considered.

This paper presents a more comprehensive and flexible method to examine the different potential sub-strategies and link them into sectoral-specific infrastructure provision strategies. The strategies have an explicit connection with policy-relevant commitments (e.g. investments and the approach to demand management and environmental issues), spanning a wide spectrum of policy instruments (from demand to technology and major investments). These sectoral strategies can further be combined to represent cross-sector strategy portfolios, developed by assessing infrastructure provision strategies from each sector which have common underlying assumptions.

Further, the method is flexible and can evolve with our developing understanding; existing portfolio members can be adapted, or new portfolio members can be added, since each strategy portfolio consists of a subset of sectoral strategy components, which can be 
changed according to the assumptions of each strategy portfolio in terms of the range of investment requirements, approaches to demand management and emphasis on environmental change mitigation.

However, there are limitations in this methodology: numerous aspects of society which could also be considered as 'infrastructure' are not included in this framework, such as riskreduction, security and defence, banking, education and health, as well as the role of the built environment. There is an assumption that the socio-economic system is not dependent on the performance of infrastructure, a feedback which this framework does not address. An important innovation of future research will be a modelling approach which includes coevolution of socio-economic and technical systems. Nevertheless, treating the economy and demography as exogenous and uncertain inputs can offer a more readily understandable approach for policy and decision analysis.

This approach is being developed and applied in ITRC's national infrastructure assessment [58]. This considers the problem of long-term planning of infrastructure services, and is testing the robustness of a range of diverse strategies (represented in the suite of sectoral models as a specified range of input variables, which in turn represent changes in capacity of and demand for infrastructure services) across a highly uncertain future (presented as a wide range of plausible future socio-economic and environmental conditions). ITRC has worked in close collaboration with a range of policy stakeholders, so that the methods are tested and validated against real-world experience and hence represent coproduced views of policy futures. The next steps within the ITRC programme are to apply the methodologies set out here to the developed suite of infrastructure models, including the development of database and visualisation tools. There will also be further investigation into the key interactions between energy and the other sectors (particularly transport and water).We expect that the methods described here could find wide application in similar analyses of systems (across a range of spatial levels) which strongly interact with complex policy choices.

\section{Acknowledgment}

All authors acknowledge funding of the work by the EPSRC (Engineering and Physical Sciences Research Council of the UK) under Program Grant EP/I01344X/1 as part of the Infrastructure Transitions Research Consortium (ITRC, www.itrc.org.uk). We also thank all ITRC colleagues for their continuing help in developing and adapting the approach to strategy generation presented in this paper. 


\section{References}

[1] CST, A national infrastructure for the 21st century, London, UK, 2009.

[2] ICE, State of the Nation: defending critical infrastructure, London, UK, 2009.

[3] HM Treasury, Infrastructure UK, National Infrastructure Plan 2010, London, UK, 2010.

[4] ICE, State of the Nation: infrastructure 2010, London, UK, 2010.

[5] Urban Land Institute and Ernst and Young, Infrastructure 2010 - investment imperative, Urban Land Institute, Washington DC, USA, 2011.

[6] Urban Land Institute and Ernst and Young, Infrastructure 2011 - a strategic priority, Urban Land Institute, Washington DC, USA, 2011.

[7] WEF, The global competitiveness report 2011-2012, Geneva, 2011.

[8] J.W. Hall, J.J. Henriques, A.J. Hickford, R.J. Nicholls, Systems-of-systems analysis of national infrastructure, Proceedings of ICE - Engineering Sustainability, 166 (2013) 249-257.

[9] J.W. Hall, J.J. Henriques, A.J. Hickford, R.J. Nicholls, P. Baruah, M. Birkin, M. Chaudry, T.P. Curtis, N. Eyre, C. Jones, C.G. Kilsby, A. Leathard, A. Lorenz, N. Malleson, F.N. McLeod, W. Powrie, J.M. Preston, N. Rai, R. Street, A. Stringfellow, C. Thoung, P. Tyler, R. Velkiene, G. Watson, J. Watson, Assessing the long-term performance of cross-sectoral strategies for national infrastructure, Journal of Infrastructure Systems, 20 (2014).

[10] A. Faber, A.M. Idenburg, H.C. Wilting, Exploring techno-economic scenarios in an inputoutput model, Futures, 39 (2007) 16-37.

[11] T. Marshall, UK Working Paper - Planning and infrastructure sectors - a history, in: Department of Planning, Oxford Brookes University (Ed.), Oxford, UK, 2010.

[12] D. Helm, J. Wardlaw, B. Caldecott, Delivering a 21st Century infrastructure for Britain, Policy Exchange, London, 2009.

[13] HM Treasury, Infrastructure UK, National Infrastructure Plan 2011, London, UK, 2011.

[14] HM Treasury, Infrastructure UK, National Infrastructure Plan: update 2012, London, UK, 2012.

[15] A. Otto, J.W. Hall, A.J. Hickford, R.J. Nicholls, D. Alderson, S. Barr, A quantified systemof-systems modelling framework for robust national infrastructure planning, IEEE Systems Journal, Accepted (2014).

[16] J. Abildtrup, E. Audsley, M. Fekete-Farkas, C. Giupponi, M. Gylling, P. Rosato, M. Rounsevell, Socio-economic scenario development for the assessment of climate change impacts on agricultural land use: a pairwise comparison approach, Environmental Science \& Policy, 9 (2006) 101-115.

[17] R.H. Moss, J.A. Edmonds, K.A. Hibbard, M.R. Manning, S.K. Rose, D.P. van Vuuren, T.R. Carter, S. Emori, M. Kainuma, T. Kram, G.A. Meehl, J.F.B. Mitchell, N. Nakicenovic, K. Riahi, S.J. Smith, R.J. Stouffer, A.M. Thomson, J.P. Weyant, T.J. Wilbanks, The next generation of scenarios for climate change research and assessment, Nature, 463 (2010) 747-756.

[18] P.D. Raskin, F. Monks, T. Ribiero, D.P. van Vuuren, M. Zurek, Global scenarios in historical perspective, in: S.R. Carpenter, P.L. Pingali, E.M. Bennett, M. Zurek (Eds.), Ecosystems and human well-being: scenarios, Island Press, Washington, D.C., USA, 2005, pp. 35-43.

[19] P. Bishop, A. Hines, T. Collins, The current state of scenario development: an overview of techniques, Foresight - The journal of future studies, strategic thinking and policy, 9 (2007) 5-25.

[20] P.W.F. van Notten, J. Rotmans, M.B.A. van Asselt, D.S. Rothman, An updated scenario typology, Futures, 35 (2003) 423-443. 
[21] M. Amer, T.U. Daim, A. Jetter, A review of scenario planning, Futures, 46 (2013) 23-40.

[22] N.W. Arnell, M.J.L. Livermore, S. Kovats, P.E. Levy, R. Nicholls, M.L. Parry, S.R. Gaffin, Climate and socio-economic scenarios for global-scale climate change impacts assessments: characterising the SRES storylines, Global Environmental Change, 14 (2004) 3-20.

[23] Foresight Futures, Foresight Futures 2020 - Revised scenarios and guidance, Department of Trade and Industry, 2001.

[24] C.R. Thorne, E.P. Evans, E.C. Penning-Rowsell, Future flooding and coastal erosion risks, Thomas Telford, London, 2007.

[25] I.P. Holman, M.D.A. Rounsevell, G. Cojacaru, S. Shackley, C. McLachlan, E. Audsley, P.M. Berry, C. Fontaine, P.A. Harrison, C. Henriques, M. Mokrech, R.J. Nicholls, K.R. Pearn, J.A. Richards, The concepts and development of a participatory regional integrated assessment tool, Climatic Change, 90 (2008) 5-30.

[26] R.J. Nicholls, R.S.J. Tol, Impacts and responses to sea-level rise: a global analysis of the SRES scenarios over the twenty-first century, Philosophical Transactions of the Royal Society A: Mathematical, Physical and Engineering Sciences, 364 (2006) 1073-1095.

[27] D. Anthoff, R. Nicholls, R.J. Tol, The economic impact of substantial sea-level rise, Mitig Adapt Strateg Glob Change, 15 (2010) 321-335.

[28] D.P. van Vuuren, M.T.J. Kok, B. Girod, P.L. Lucas, B. de Vries, Scenarios in Global Environmental Assessments: Key characteristics and lessons for future use, Global Environmental Change, 22 (2012) 884-895.

[29] L. Borjeson, M. Hojer, K. Dreborg, T. Ekvall, G. Finnveden, Scenario types and techniques: Towards a user's guide, Futures, 38 (2006) 723-739.

[30] F. Berkhout, J. Hertin, A. Jordan, Socio-economic futures in climate change impact assessment: using scenarios as 'learning machines', Global Environmental Change, 12 (2002) 83-95.

[31] R.J. Lempert, S.W. Popper, S.C. Bankes, Shaping the next one hundred years: New methods for quantitative, long-term policy analysis, RAND Corporation, 2003.

[32] M. Chaudry, N. Jenkins, M. Qadrdan, J. Wu, Combined gas and electricity network expansion planning, Applied Energy, 113 (2014) 1171-1187.

[33] P. Baruah, N. Eyre, Simulation of residential energy demand in Great Britain under a range of demographic change and energy system transition pathways, In preparation for Energy Policy, (2014).

[34] S.P. Blainey, J.M. Preston, F.N. McLeod, A long term capacity and demand assessment model for the UK transport system, in: European Transport Conference, Glasgow, October, 2012.

[35] J.W. Hall, J.J. Henriques, A.J. Hickford, R.J. Nicholls, (eds), A Fast Track Analysis of strategies for infrastructure provision in Great Britain: Technical report, Environmental Change Institute, University of Oxford, 2012.

[36] R.J. Lempert, A new decision sciences for complex systems, Proceedings of the National Academy of Sciences of the United States of America, 99 (2002) 7309-7313.

[37] C. Zuo, M. Birkin, N. Malleson, Dynamic microsimulation modelling for national infrastructure demand in an uncertain future, in: GeoComputation 2013, Wuhan, China, 2013.

[38] ONS, National population projections, 2010-based statistical bulletin, in: Office for National Statistics (Ed.), 2010.

[39] E.M. Gramlich, Infrastructure investment: a review essay, Journal of Economic Literature, 32 (1994) 1176-1196. 
[40] S.-H.P. Lau, C.-Y. Sin, Public infrastructure and economic growth: time-series properties and evidence, Economic Record, 73 (1997) 125-135.

[41] B. Égert, T. Kozluk, D. Sutherland, Infrastructure and growth: empirical evidence, 2009.

[42] B. Sanchez-Robles, Infrastructure investment and growth: some empirical evidence, Contemporary Economic Policy, 16 (1998) 98-108.

[43] R.B. Street, A. Steynor, P. Bowyer, K. Humphrey, Delivering and using the UK climate projections 2009, Weather, 64 (2009) 227-231.

[44] IPCC, Climate Change 2007: Working Group III: Mitigation of climate change, 2007.

[45] IPCC, Special Report on Emissions Scenarios: A Special Report of Working Group III of the Intergovernmental Panel on Climate Change, IPCC, Geneva, Switzerland, 2000.

[46] D.P. van Vuuren, J. Edmonds, M. Kainuma, K. Riahi, A. Thomson, K. Hibbard, G.C. Hurtt, T. Kram, V. Krey, J.-F. Lamarque, T. Masui, M. Meinshausen, N. Nakicenovic, S.J. Smith, S.K. Rose, The representative concentration pathways: an overview, Climatic Change, 109 (2011) 5-31.

[47] E. Kriegler, B.C. O’Neill, S. Hallegatte, T. Kram, R.J. Lempert, R.H. Moss, T. Wilbanks, The need for and use of socio-economic scenarios for climate change analysis: A new approach based on shared socio-economic pathways, Global Environmental Change, 22 (2012) 807822.

[48] IPCC, Climate Change 2013: The Physical Science Basis. Working Group I contrbution to the Intergovernmental Panel on Climate Change Fifth Assessment Report (AR5), IPCC, Geneva, Switzerland, 2013.

[49] S.P. Blainey, A.J. Hickford, J.M. Preston, Planning transport networks for an uncertain future: a UK case study, in: European Transport Conference 2013, Frankfurt, Germany, 2013.

[50] C. Brand, M. Tran, J. Anable, The UK transport carbon model: An integrated life cycle approach to explore low carbon futures, Energy Policy, 41 (2012) 107-124.

[51] A. Schafer, L. Dray, E. Andersson, M.E. Ben-Akiva, M. Berg, K. Boulouchos, P. Dietrich, O. Froidh, W. Graham, R. Kok, S. Majer, B. Nelldal, F. Noembrini, A. Odoni, I. Pagoni, A. Perimenis, V. Psaraki, A. Rahman, S. Safarinova, M. Vera-Morales, TOSCA Project Final Report: Description of the main S\&T results/foregrounds, 2011.

[52] N. Hill, M. Morris, I. Skinner, SULTAN: Development of an illustrative scenarios tool for assessing potential impacts of measures on EU transport GHG, 2010.

[53] Committee on Climate Change, The fourth carbon budget - reducing emissions through the 2020s, London, UK, 2010.

[54] McKinsey \& Company, Roads toward a low-carbon future: Reducing CO2 emissions from passenger vehicles in the global road transportation system, McKinsey \& Company, New York, 2009.

[55] M. Page, C. Kelly, A. Bristow, Exploring scenarios to 2050 for hydrogen use in transport in the UK, in: European Transport Conference, Strasbourg, 2004.

[56] I. Skinner, H. van Essen, R. Smokers, N. Hill, Towards the decarbonisation of the EU's transport sector by 2050, 2010.

[57] R.A. Kelly, A.J. Jakeman, O. Barreteau, M.E. Borsuk, S. ElSawah, S.H. Hamilton, H.J. Henriksen, S. Kuikka, H.R. Maier, A.E. Rizzoli, H. van Delden, A.A. Voinov, Selecting among five common modelling approaches for integrated environmental assessment and management, Environmental Modelling \& Software, 47 (2013) 159-181.

[58] J.W. Hall, M. Tran, A.J. Hickford, R.J. Nicholls, A. Otto, Planning Infrastructure for the 21st Century, Cambridge University Press, Cambridge, 2014. 